\title{
REFLECTIONS
}

\section{Within minutes from Mseleni to Cape Town ...}

\author{
A K Nelson, $\mathrm{MB} \mathrm{ChB}$ \\ Corresponding author: A K Nelson (aurelie.nelson@gmail.com; msfocb-khayelitsha-eid@brussels.msf.org) \\ Dr Aurélie Nelson is the Early Infant Diagnosis Project Manager for Médecins Sans Frontières, Khayelitsha, Cape Town, South Africa
}

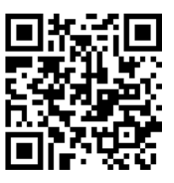

'It's impossible to roll out antiretrovirals in Africa!' I clearly remember hearing this on a daily basis while studying in Canada 13 years ago. And there were strong points made: too expensive, not enough doctors, risk of resistance, how to monitor patients in resource-limited settings, and so forth. Thankfully, not everyone believed this and we advocated and pressured from all around the world, and the price of antiretroviral therapy (ART) did come down, by more than ten times.

\section{‘... we advocated and pressured from all around the world, and the price of ART did come down, by more than ten times.'}

Ten years later, after studying medicine in the United Kingdom (UK), I moved to Mseleni Hospital in northern KwaZulu-Natal for my 'voluntary community service'. The medical director of the hospital had been there for more than 30 years and started rolling out ART unlawfully before March 2004. He had developed some outreach clinics and managed to have his hospital very well staffed with local and international doctors. With some basic teaching in HIV and tuberculosis (TB), we (the UK doctors) were very soon thrown into the deep end. The first three months of practising were a steep learning curve, particularly in HIV medicine. We were working in the outpatient department where AIDS patients often presented very late, and on the ward where we had very complex co-infected TB/HIV patients with poor referral systems. The mortality due to HIV/TB was often very high. Despite some HIV-positive patients still presenting very late (particularly men), we had good statistics on testing people and good prevention of mother-to-child transmission (PMTCT) coverage.
As part of my duties, I was visiting an outreach clinic weekly called Manaba Clinic. Most of my work there was to initiate patients on ART and to follow up problematic cases. I recall the particular case of a 9-year-old boy who had virological failure on ART, despite adherence reinforcement. After only a few months of working with HIV and ART, and without access to resistance testing, I felt quite out of my depth. Extraordinarily, however, I was able to get advice from an HIV specialist paediatrician in Cape Town within minutes via the national HIV hotline. Following his advice, I started the little boy on lamivudine (3TC) monotherapy as a bridging measure, while waiting for new paediatric formulations to be made available.

A few months later, I saw the same 9-year-old boy and his mother for a follow-up appointment. His viral load was now nearly suppressed and I was going to continue the 3TC monotherapy as per the advice I had received, when mum (via a translator) started objecting. She had always been told that her child must take three antiretrovirals, so why was he taking only one drug? Wasn't there a risk of resistance?

\section{'She had always been told that her child must take three antiretrovirals, so why was he taking only one drug? Wasn't there a risk of resistance?'}

She made me realise how far we had come in ten years: ART was being rolled out to places hundreds of kilometres from the nearest city; patients with basic education were gaining a good understanding of a complex disease; and expert help from thousands of kilometres away was available within minutes over the phone. Against all odds, what a long way HIV treatment had come!

S Afr J HIV Med 2014;15(1):13. DOI:10.7196/SAJHIVMED.1010 